\title{
DEVELOPMENT OF AN ALL-PURPOSE FREE PHOTOGRAMMETRIC TOOL
}

\author{
D. González-Aguilera ${ }^{1} *$, L. López-Fernández ${ }^{1}$, P. Rodriguez-Gonzalvez ${ }^{1}$, \\ D. Guerrero ${ }^{2}$, D. Hernandez-Lopez ${ }^{2}$ \\ F. Remondino ${ }^{3}$, F. Menna ${ }^{3}$, E. Nocerino ${ }^{3}$, I. Toschi ${ }^{3}$ \\ A. Ballabeni ${ }^{4}$, M. Gaiani ${ }^{4}$ \\ ${ }^{1}$ TIDOP Research Unit, University of Salamanca, USAL, Spain \\ (daguilera, luisloez89,pablorgsf)@usal.es, http://tidop.usal.es \\ ${ }^{2}$ University of Castilla-La Mancha, UCLM, Spain \\ (dguerrero, david.hernandez)@uclm.es \\ ${ }^{3}$ 3D Optical Metrology (3DOM) unit, Bruno Kessler Foundation (FBK), Trento, Italy \\ (remondino, fmenna, nocerino, toschi)@fbk.eu, http://3dom.fbk.eu \\ ${ }^{4}$ Dept. of Architecture, University of Bologna, University of Bologna, Italy \\ (andrea.ballabeni, marco.gaiani)@ unibo.it
}

Commission VI - WG 2

KEY WORDS: Photogrammetry, Free Software, Feature Extraction and Matching, Camera Calibration, Image Orientation, Dense Matching, 3D reconstruction, Computer aided-teaching (CAT), E-learning

\begin{abstract}
:
Photogrammetry is currently facing some challenges and changes mainly related to automation, ubiquitous processing and variety of applications. Within an ISPRS Scientific Initiative a team of researchers from USAL, UCLM, FBK and UNIBO have developed an open photogrammetric tool, called GRAPHOS (inteGRAted PHOtogrammetric Suite). GRAPHOS allows to obtain dense and metric 3D point clouds from terrestrial and UAV images. It encloses robust photogrammetric and computer vision algorithms with the following aims: (i) increase automation, allowing to get dense 3D point clouds through a friendly and easy-to-use interface; (ii) increase flexibility, working with any type of images, scenarios and cameras; (iii) improve quality, guaranteeing high accuracy and resolution; (iv) preserve photogrammetric reliability and repeatability. Last but not least, GRAPHOS has also an educational component reinforced with some didactical explanations about algorithms and their performance. The developments were carried out at different levels: GUI realization, image pre-processing, photogrammetric processing with weight parameters, dataset creation and system evaluation.

The paper will present in detail the developments of GRAPHOS with all its photogrammetric components and the evaluation analyses based on various image datasets. GRAPHOS is distributed for free for research and educational needs.
\end{abstract}

\section{INTRODUCTION}

Photogrammetry is nowadays facing new challenges and changes particularly related to automation, ubiquitous processing, variety of datasets and applications. In the last years the scientific community has replied with new algorithms and methodologies and various commercial or free software are now available, often as one-click solution. Performances, reliability, transparency, repeatability, accuracy potential and usability vary a lot according to packages, applications and users (see https://en.wikipedia.org/wiki/Comparison_of_photogrammetry_ software for a quite complete list of tools). With the aim to deliver a free image processing package able to fulfil all photogrammetric requirements, a team of researchers developed the GRAPHOS tool. The main goal of the GRAPHOS project, supported by an ISPRS Scientific Initiative, was to realize an allpurpose open-source photogrammetric platform mainly for terrestrial and UAV applications. GRAPHOS aims to bring photogrammetry and computer vision even more closer realizing a tool which integrates different algorithms and methodologies for automated image orientation and dense 3D reconstruction from set of unordered images. Automation was not the only keydriver of the tool but precise processing, reliability, repeatability and guidelines for non-experts were also considered. The tool wants to provide an educational, easy-to-use and ease-to learn framework for image-based 3D reconstruction applied to architectural, heritage and engineering applications.

Since the photogrammetric process involves basically three different steps (e.g. extraction and matching of image correspondences, camera calibration and image orientation, dense point cloud generation), the specifics goals of GRAPHOS are:

1. Provide image pre-processing algorithms and strategies for improving the image quality and thus the photogrammetric processes. In particular, "Contrast Preserving Decolorization" and "Wallis filtering" were implemented. In addition, an automatic estimation of sensor size parameters was included for non-expert users and unknown cameras.

2. Incorporate various tie-point detectors and descriptors to improve the image matching phase, paying particular attention to textureless and repeated pattern situations. In particular, SIFT (Lowe, 1999), ASIFT (Morel and Yu, 2009) and MSD (Tombari and Di Stefano, 2014) detectors were combined with SIFT descriptor in order to provide the best keypoint extractor. Furthermore, three different pair-

\footnotetext{
* Corresponding author
} 
wise feature-based matching approaches were used: from the classical L2-Norm (Lucas and Kanade, 1981) and the efficient FLANN (Muja and Lowe, 2009) matcher to a more sophisticated one based on a sequential robust matcher strategy.

3. Improve the computational cost exploiting GPU or parallel computing. In particular, CUDA programming capabilities were included in some steps in order to improve computation times, especially in dense matching; whereas parallelizing was used in image pre-processing and feature extraction.

4. Improve the bundle adjustment performances using an optimization of tie-points distribution and different selfcalibration approaches (by varying the number of computed additional parameters and allows the user to fix/weight them).

5. Improve the dense matching methods with multi-view approaches in order to increase the reliability and quality of the $3 \mathrm{D}$ point cloud.

GRAPHOS has been led and managed by USAL in collaboration with UCLM, FBK and UNIBO. The secret of success was to find an efficient and robust pipeline for automated image orientation and dense 3D reconstruction, notwithstanding important aspects like self-calibration with various additional parameters, weight for observations/unknown parameters, reliability, statistics, etc. This paper has been structured as follows: after this Introduction and the following short state-of-the-art in image-based 3D reconstruction, Section 2 remarks the educational and transversal component of GRAPHOS; Section 3 describes the created datasets together with the developed workflow; Section 4 outlines some experimental results, analysing also computational costs. A final section is devoted to report conclusions and future ideas.

\subsection{State of the art in image-based 3D reconstruction}

In the last decade the image-based pipeline based on photogrammetric and computer vision algorithms for $3 \mathrm{D}$ reconstruction purposes has become a powerful and valuable approach - normally called Structure from Motion (SfM) - in many applications and fields. Indeed it generally ensures sufficient automation, efficient results and ease of use, even for non-professional. The recent progresses were achieved in all core components of the image-based pipeline: image pre-processing (Maini and Aggarwal, 2010; Verhoeven et al., 2015), tie-point extraction (Apollonio et al., 2014; Hartmann et al., 2015), bundle adjustment (Agarwal et al., 2010; Wu et al., 2011) and dense and precise points clouds generation (Remondino et al., 2014). These progresses have led to fully automated methodologies able to process large image datasets and deliver $3 \mathrm{D}$ results with a level of detail and precision variable according to the applications (Snavely et al., 2008; Frahm et al., 2010; Crandall et al., 2013). Particularly in terrestrial applications, the level of automation is reaching very high standards and it is increasing the impression that few randomly acquired images - even found on the Internet (Heinly et al., 2015) - and a black-box tool are sufficient to produce a metrically precise $3 \mathrm{D}$ point cloud or textured $3 \mathrm{D}$ model. But when it comes to applications different from web visualization or quick and funny $3 \mathrm{D}$ reconstructions, end-users are still missing a valuable solution for metric applications where results can be deeply analysed in terms of accuracy and reliability. As a result, algorithms and methods could be understated, being able to identify errors, weakness in dataset or potentials for its improvement. In this context, some years ago photogrammetric educational tools were developed, like sv3DVision (González-Aguilera and Gomez Lahoz, 2006) which allows to perform a dimensional analysis of engineering and architectural scenes or 3D reconstructions using a single view acquired with an unknown camera. In addition, a simulator interface based on artificial images is offered to introduce and modify internal and external orientation parameters together with a simulation of random errors, analysing precision and reliability parameters. Didactical tools based on multiple images were developed too, like Arpenteur (Gussenmayer and Drap, 2001) and PW-Photogrammetry Workbench (Gonzalez-Aguilera et al., 2012; Zancajo-Blazquez et al., 2015). The former was a web application for architectural photogrammetry, whereas the latter represents a real low-cost alternative to laser scanning systems that allows automatism and flexibility in the management of any block of images (from vertical stereo to convergent oblique) and any type of camera (calibrated or non-calibrated). More recently, PhoX was developed in order to provide a tool for self-learning, test and exercise with real photogrammetric data (Luhmann, 2016). The main purpose of this software is data analysis, interactive image measurements, simulation and visualization of results.

\section{A NEW EDUCATIONAL APPROACH TO GEOMATICS}

Reality-based 3D models have become an essential part of many applications for architects and civil engineers ranging from documentation, digital restoration, visualization, inspection, planning, AR/VR in the field of Architectural Heritage conservation and design or simply in the as-built documentation of bridges, roads and tunnels. In the last years capturing accurate and detailed geometric models of real world objects has become a common process. However, until today this activity was a specific task addressed to few highly skilled operators. Tools such as total stations, laser scanners and structured lighting systems are often costly, time consuming, difficult to carry and use, and they mainly require a specialized operator, as well as the first generation of image-based modeling digital tools. In recent years, a new generation of automated image-based modelling techniques combining image analysis, photogrammetry and computer vision have emerged, allowing an accurate and efficient derivation of 3D information. The increasing level of automation of these tools allows practitioners to accomplish various tasks, such as the documentation of the actual state of an artifact, even without specific skills in geomatics and computer science fields, at low cost and, at a low level, just using automated process like a black box: you give an input (a set of images) and obtain an output (a textured 3D model) without having information on what kind of processing is currently running, and which are the algorithms that underline the process (e.g. camera calibration and image orientation, dense cloud reconstruction). This is a great improvement over the previous processing tools which were tailored only for skilled operators but implies the need of knowledge inference in a new way to overcome the inability to recognize their effectiveness and nature. Overall, this new scenario involves a strong need for changing the educational processes that cannot be limited to a simple change of how knowledge is taught. Today, training techniques usually are strictly linked to traditional methods, as they are based on theoretical lectures and followed by a short experimental phase to test the theory understanding. This problem is increased by the introduction of digital and automatic tools that do not allow the development of typical sensitiveness acquired by their direct use. A central feature of the considered system is that digital photogrammetry knowledge cannot be separated from the user ability to handle specific software. This characterizes the knowledge to be taught as theoretical and practical knowledge. This, however, poses major problems to the didactic 
transposition of knowledge which must manage the relationship between theoretical knowledge and practical knowledge. Where the knowledge to be taught were perceived by teachers and students merely as expertise in the use of the software, e.g., the knowledge taught would result in mere practical knowledge but not necessarily a theoretical-practical knowledge and this, evidently, is not a desirable outcome. In this case, the acquired knowledge may be inadequate to the solution of more complex problems or in a non-standard software use. In other words, the expected knowledge is, in our case, a practical knowledge, i.e. knowledge and know-how similar to certain enduring structures of thought and action that can be activated at different times and contexts. It is not only procedural learning, useful to play some codified procedures in response to standard tasks, but also the ability to coordinate declarative knowledge, procedural knowledge and internal provisions such as motivational and affective functions in the presence of challenging situations. These learnings, classified as 'skills', reveal the different logical Our new tool GRAPHOS allows to fill the existing gap, improving the knowledge of automation processes in imagebased modelling to various users for those careers that require images as input data. In particular, it allows to introduce experimental and inductive techniques to teach image-based modelling based for documentation, virtual reality and augmented reality needs. In addition, the software allows to "universalize" the workflow from 2D (images) to 3D (models) from any image acquired in any situation by non-expert users.

\section{MATERIALS AND METHODS}

\subsection{Dataset description}

A specific database was created and used to test the capabilities and scope of GRAPHOS. Although different case studies and geometric configurations were considered, all sets of images were focused on close-range applications since GRAPHOS was created to be applied in different application fields. Additionally, smartphone and tablet sensors were tested in order to assess the flexibility of the tool in data acquisition. As a result, the database contains 38 case studies (Figure 1 and Table 1). Some case studies included a ground truth by means of control points, check points and laser scanning point cloud.

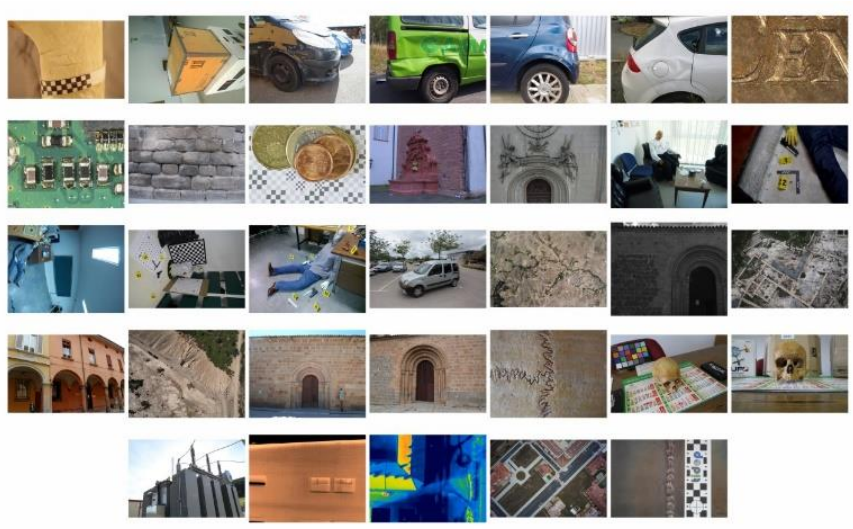

Figure 1. Some of the images of the database used in the testing phase.

\subsection{Methods}

The following figure (Figure 2) outlines the proposed pipeline (main GRAPHO) including the different implemented algorithms and processes.

\begin{tabular}{|c|c|c|c|c|}
\hline Case & Difficulty & $\begin{array}{c}\mathbf{N}^{0} \text { of } \\
\text { images }\end{array}$ & $\begin{array}{l}\text { Size } \\
(\mathbf{M p})\end{array}$ & Sensor \\
\hline Forensic & medium & 12 & 15.1 & $\begin{array}{c}\text { Canon } \\
500 \mathrm{D}\end{array}$ \\
\hline Automotive & high & 5 & 5.0 & $\begin{array}{l}\text { Lumia } \\
1020^{*}\end{array}$ \\
\hline Architecture & medium & 4 & 10.0 & Nikon D80 \\
\hline Architecture & medium & 11 & 6.3 & EOS D60 \\
\hline Forensic & very high & 23 & 12.2 & E-PM1 \\
\hline Forensic & high & 15 & 8.0 & XperiaL* \\
\hline Automotive & high & 48 & 21.0 & $\begin{array}{c}\text { EOS 5D } \\
\text { mkII }\end{array}$ \\
\hline $\begin{array}{c}\text { Aerial } \\
\text { archaeology }\end{array}$ & medium & 5 & 195.8 & $\begin{array}{c}\text { UltraCamX } \\
\text { p }\end{array}$ \\
\hline Others & very high & 30 & 1.3 & MCA6** \\
\hline $\begin{array}{c}\text { Aerial } \\
\text { archaeology }\end{array}$ & low & 58 & 21.0 & $\begin{array}{c}\text { EOS 5D } \\
\text { mkII }\end{array}$ \\
\hline $\begin{array}{c}\text { Aerial } \\
\text { engineering }\end{array}$ & low & 27 & 12.2 & E-P1 \\
\hline Forensic & low & 15 & 15.9 & E-PM2 \\
\hline Engineering & very high & 24 & 15.1 & $\begin{array}{l}\text { Canon } \\
500 \mathrm{D}\end{array}$ \\
\hline Others & very high & 7 & 0.3 & $\begin{array}{c}\text { FLIR } \\
\text { SC655** }\end{array}$ \\
\hline Urban aerial & low & 21 & 16.0 & NEX-5R \\
\hline Engineering & low & 25 & 15.1 & $\begin{array}{l}\text { Canon } \\
500 \mathrm{D} \\
\end{array}$ \\
\hline Urban aerial & low & 9 & 24.4 & Nikon D3x \\
\hline Architecture & very low & 26 & 14.6 & Canon G10 \\
\hline
\end{tabular}

Table 1. Information about some cases studies, including information on the level of difficulty (from a geometric and/or radiometric point of view) and the sensors used for data acquisition. * refers to smartphones and ** to thermographic or multispectral cameras.

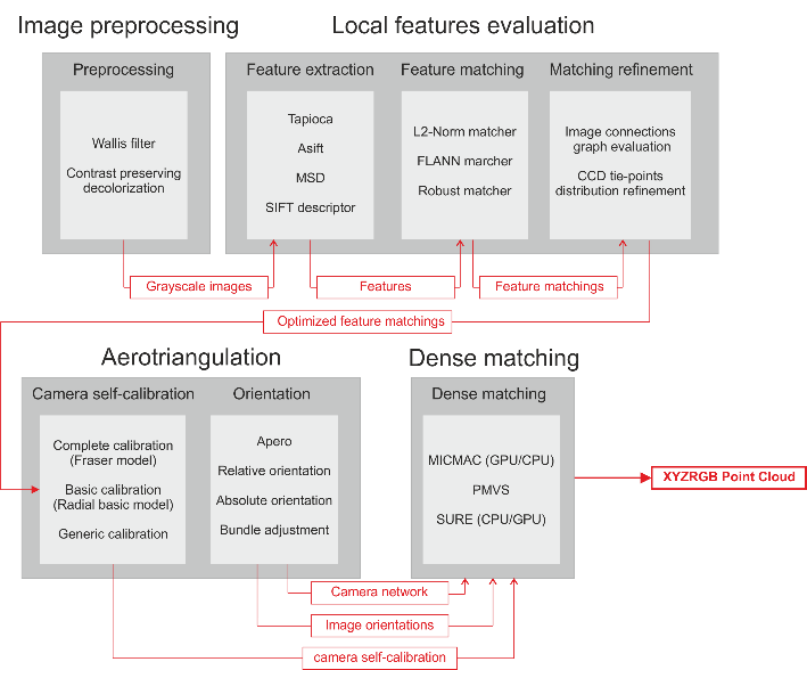

Figure 2. GRAPHOS workflow, from image pre-processing to dense point cloud generation.

3.2.1 Image acquisition protocol. Concerning the photogrammetric procedure, one of the greatest barriers for nonexpert users is the data (image) acquisition. However, whilst it may be technically simple, a good protocol requires several rules (e.g. geometrical and radiometric restrictions or camera calibration). In this sense, some basic rules have been created to support non-expert users in acquiring a good set of images under a good image network for the creation of a $3 \mathrm{D}$ object/scene (through conventional cameras and even smartphones). Although many processing packages ingest any kind of image network, the 
quality of the results can be improved by following simple protocols (Nony et al., 2012).

There are two protocols which can be used by the user:

1. Parallel protocol: it is ideal for detailed reconstructions in specific areas of an object. In this case, the user needs to capture around 5-10 images following a cross shape (Figure 3 , left). The overlap between images needs to be at least $80 \%$. The master or central image (red footprint in the figure) will entirely capture the area of interest. The other images have a complementary nature and should be taken from left, right (purple footprint), top and bottom (green footprint) with respect to the central image. These photos should adopt a certain degree of perspective, turning the camera towards the middle of the interest area, but still capturing the whole area of interest. In order to stress this parallel protocol, GRAPHOS includes a didactical tutorial for performing this type of image acquisition (https://vimeo.com/145839757).

2. Convergent protocol: it is ideal for the reconstruction of a $360^{\circ}$ object (e.g. statue, column, etc.). In this case, the user should capture the images following a ring path (keeping an approximate constant distance from the object). It is necessary to ensure a good overlap between images $(>80 \%)$ (Figure 3, right). In the situations where the object cannot be captured with a unique ring it is possible to adopt a similar procedure based on multiple rings or a half ring. Again, in order to better show this convergent protocol, GRAPHOS includes a didactical tutorial for performing this type of image acquisition (https://vimeo.com/127157351).
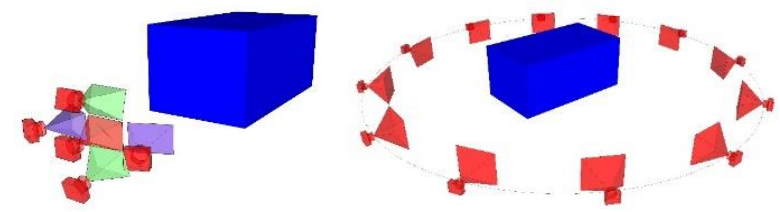

Figure 3. Different acquisition protocols suggested in GRAPHOS. Parallel protocol (left) and convergent protocol (right).

Las but not least, trying to make the data acquisition more simple, GRAPHOS allows to cope with video files, so both protocols could be reproduced recording a video. This approach is not advisable in those cases where accuracy and resolution is required since the processing of video sequence depicts images of lower resolution and quality.

The protocols are of course highly suggested - but not mandatory - in order to improve the quality of the results and facilitate processing algorithms.

3.2.2 Image pre-processing. The image pre-processing is an important step since it can provide better feature extraction and matching results, in particular in those cases where the texture quality is unfavourable. Two pre-processing functions are available in GRAPHOS:

1. Contrast Preserving Decolorization: it applies a decolorization (i.e. RGB to Gray) to the images preserving contrast (Lu et al., 2012). In particular, a bimodal distribution to constrain spatial pixel difference and for automatic selection of suitable gray scale has been included in order to preserve the original contrast. Contrary to other methods, it delivers better images for the successive feature extraction and matching steps (Gaiani et al., 2016).

2. Wallis filter: this enhancement algorithm (Wallis, 1974) is available for those textureless images or images with homogeneous texture, improving the keypoint extraction and matching steps. In particular, the Wallis filter adjusts brightness and contrast of the pixels that lie in certain areas where it is necessary, according to a weighted average. As a result, the filter provides a weighted combination of the average and the standard deviation of the original image. Although default parameters are defined in GRAPHOS, the average contrast, brightness, standard deviation and window size can be introduced by the user (as advanced parameters) in case the default values are not suitable.

Both pre-processing strategies are available in GRAPHOS with didactical material in order to reinforce not only the application but also the knowledge about these algorithms.

3.2.3 Feature extraction and matching. In typical close-range photogrammetric cases, feature extraction and matching encloses two main issues: (i) computational cost and (ii) important radiometric and geometric variations. For the former, GRAPHOS allows to work with different resampling levels (image downscaling to $15 \%, 30 \%$ and $50 \%$ of the original image resolution), especially in those cases with a huge number of images. Regarding to the latter issue, GRAPHOS includes three different detectors/descriptors that can be combined with three different matching strategies in order to find the best correspondences (in terms of quality and distribution) between images:

1. Tapioca: it is a combined keypoint detector and descriptor (Pierrot-Deseilligny et al., 2015). The keypoint detection and description strategy is based on the SIFT algorithm developed by (Lowe, 1999).

2. ASIFT: it is a keypoint detector developed by (Morel and $\mathrm{Yu}, 2009)$. ASIFT considers two additional parameters that control the presence of images with different scales and rotations. In this manner, the ASIFT algorithm can cope with images displaying a high scale and rotation difference, common in oblique scenes. The result is an invariant algorithm that better support changes in scale, rotations and movements between images. The description strategy is based on SIFT method.

3. MSD: it is a keypoint detector developed by Tombari and Di Stefano (2014) which finds maximal self-dissimilarities. In particular, it supports the hypothesis that image patches highly dissimilar over a relatively large extent of their surroundings hold the property of being repeatable and distinctive. The description strategy is based on SIFT method.

Once the keypoints are extracted, a pairwise matching strategy should be applied to identify the correct image correspondences. GRAPHOS contains three different matching strategies, independently from the protocol followed in image acquisition:

1. Tapioca: the matching strategy of this method applies a strategy based on the L2-Norm to find the point with the closest descriptor for each keypoint extracted in the considered image. Tapioca allows two select tree strategies: (i) for small unordered image datasets, the keypoint matching is recursively calculated for all possible image pairs; (ii) for huge unordered datasets, the keypoint matching is calculated in two consecutive steps, the first computes the images connection graph at a low resolution and the second perform the high-resolution feature extraction and matching following the prior calculated connection graph; (iii) for linearly structured image datasets, the matching strategy can check only between $n$ adjacent images.

2. Robust matcher approach: a brute force matching strategy is used in a two-fold process: (i) first, for each extracted point the distance ratio between the two best candidates in the other image is compared with a threshold; (ii) second, those remaining pairs of candidates are filtered by a threshold which expresses the discrepancy between 
descriptors. As result, the final set of matching points is used to compute the relative orientation (fundamental matrix) between both images, re-computing the process in order to achieve optimal results. Additionally, and if the number of matched points is high, a filtering based on the $n$-best according to their quality ranking can be applied, making easy and more robust the next step of self-calibration and orientation.

3. FLANN: this is an optimal and alternative method (Muja and Lowe, 2009) useful when the number of extracted keypoints is pretty high. It is similar to the previous method but it optimizes the computational time.

A special tutorial about feature based matching (FBM) is included in GRAPHOS to support this step.

3.2.4 Relative orientation and self-calibration. Since the feature extraction and matching usually provides a high number of points, GRAPHOS includes an optimization step where only the most salient tie points are preserved. In this way the relative orientation and self-calibration steps can be optimized (in terms of quality and computational time). In particular, the optimization method requires three different parameters: (i) number of rows and columns for a cell $(128 \times 128,64 \times 64,32 \times 32,16 \times 16,8 \times 8)$, so the image is divided in cells according to this size; (ii) number of tie points per cell $(1,2,5,10,20,50,100)$; (iii) precision threshold for tie points in those cases where can be possible to assess tie points quality.

Once the tie points have been optimized (reduced), the image orientation is performed through a combination between computer vision and photogrammetric strategies included in the open-source tool Apero (Deseilligny and Clery, 2011). This combination is fed by the previously extracted and optimized tie points. In a first step, an approximation of the external orientation of the cameras is calculated following a fundamental matrix approach. Later, everything is refined by a bundle adjustment solution based on Gauss-Newton method. During the processing, the user can also run a self-calibration to simultaneously compute also the interior camera parameters. GRAPHOS allows to use three different types of self-calibration modes:

1. Basic calibration: five interior parameters (f-focal length, xo,yo-principal point of symmetry, $k_{1}, k_{2}$-radial distortion) are used (Kukelova and Pajdla, 2007). This model is suitable when using unknown cameras or with cameras with low quality (such as smartphones or tablets) or when the image network is not very suitable for camera calibration.

2. Complete calibration: it includes 12 interior parameters $(f$, $x_{0}, y_{0}, x_{1}, y_{1}-$ distortion center, $k_{1}, k_{2}, k_{3}, p_{1}, p_{2}$, scaling and affinity factors) (Fraser, 1980).

3. Advanced calibration: it allows the user to select which interior parameter should be computed, fixing or unfixing them in the previous calibration modes.

The results of the bundle adjustment are available in detail in a $\log$ file, while image orientation results are graphically visible in the form of a sparse point cloud and image pyramids.

GRAPHOS includes some routines to export these orientation results to dense matching tools such as SURE (Rothermel et al., 2012) and PMVS (Furukawa and Ponce, 2010).

Additionally, from an educational and research point of view, GRAPHOS includes different well-known radial distortion profiles (i.e. Gaussian, Balanced) together with a converter to export the calibration results to the most common close-range commercial tools such as PhotoScan and PhotoModeler. As a result, different comparisons and analysis can be carried out through different tools.

3.2.5 Absolute orientation and tuning of photogrammetric block and self-calibration. GRAPHOS allows to refine / re-run the bundle adjustment employing ground control points (GCP) or coded scale bars (CSB). In case of CSB, we have designed specific coded targets which can be automatically recognized by the software, establishing a scaling procedure without the influence of the user's skill and thus obtaining a 3D point cloud with metric properties. Through this process a better adjustment is obtained, avoiding errors that would be obtained and propagated using a solid rigid transformation. Regarding camera calibration refinement, the user can select those internal parameters that will be refined and those which remain fixed. This step is supported in GRAPHOS through a didactical simulator which allows a user to design and adjust a photogrammetric network (estimating its accuracy and reliability).

3.2.6 Dense matching. One of the recent greatest breakthroughs in photogrammetry was the exploitation, from a geometric point of view, of the image spatial resolution (size in pixels). This allowed to obtain a 3D object point of each image pixel. Different strategies, stereo or multi-view, have emerged in the recent years (Remondino et al., 2014), such as the Semi-Global Matching (SGM) approach which is the actual state-of-the-art method. Most of these strategies, fed by the camera parameters and complemented by the epipolar geometry, are focused on the minimization of an energy function. Multi-view approaches are incorporated in GRAPHOS in order to increase the reliability of the $3 \mathrm{D}$ results and to better cope with the case of complex study cases. GRAPHOS offers two different dense matching approaches:

1. MicMac algorithm (Pierrot-Deseilligny et al., 2015), ideal for parallel protocols.

2. PMVS algorithm (Furukawa and Ponce, 2010), more suited for convergent protocols and which allows a complete reconstruction of the scene.

For those users who owns a licence of SURE, a wrapper interface is available to launch the matching algorithm. SURE can be included in the workflow as an optimal and more efficient tool both for parallel and oblique protocols.

GRAPHOS includes some export functionalities (available with advanced parameters) in order to work with various point clouds formats (e.g. LAS, LAZ, PLY, etc.).

From an educational point of view, GRAPHOS includes a SGM tutorial which outlines the main idea about the dense matching strategy, to reveal important photogrammetric notions students and researchers.

\section{EXPERIMENTAL RESULTS}

\subsection{Image pre-processing influence in feature extraction and} matching process

The use of the correct image pre-processing algorithm is the basis for a successful processing of an image dataset. For those cases with homogeneous texture or textureless surfaces, the use of Wallis filter improves significantly the number and spatial distribution of features extracted by the keypoint detector (Figure 4) optimizing the chances of success in the relative orientation and self-calibration process.

4.2 Image connections refinement through graph evaluation When the feature matching is computed, it is possible to evaluate the image connectivity to reject wrong image pairs due to presence of outliers during the keypoint detection phase. This analysis is based in the bidirectional analysis of the homography related for each pair of linked images. 

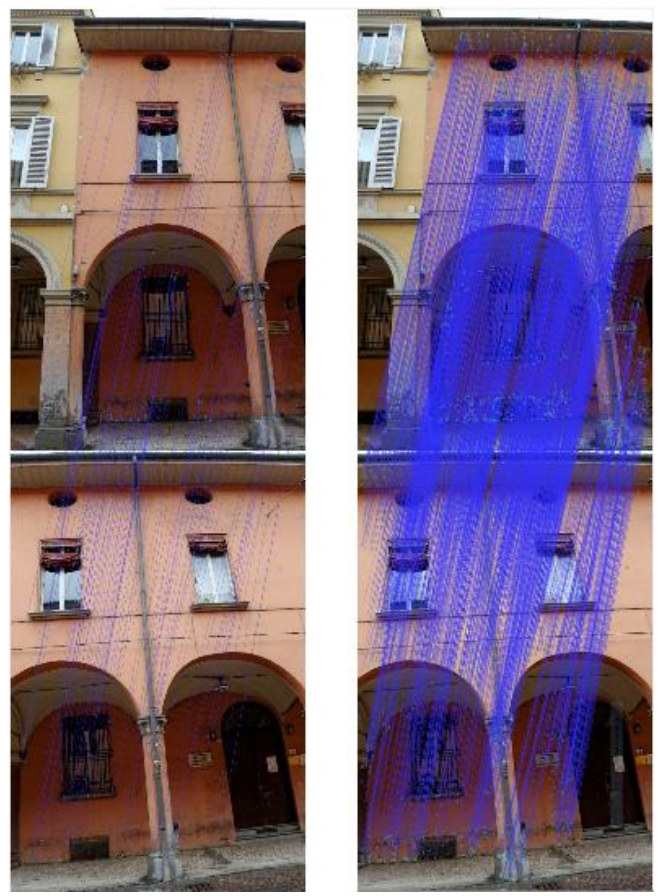

Figure 4. Tapioca feature extraction and matching results with different image pre-processing algorithms. Contrast preserving decolorization -

111 matches (left) and Wallis filter - 1814 matches (right).

In this way, an efficiency analysis of keypoints can be performed. For any image pair, fundamental matrix is computed by means of a least squares minimization and a pre-established number of iterations, unless the numbers of points is insufficient to provide a solution, in which case it applies the 8-points algorithm. Then, the homography is computed and applied over the image perimeter, to re-project on the neighbor image. Once done, these geometrical parameters are evaluated according to preestablished thresholds: the area of the re-projected image on the neighbor image, the area of the intersected polygons and the mean distance among the polygon vertices are considered. All these parameters are evaluated relatively to the image size. In those cases that the above conditions are not fulfilled, the connection is tagged as not valid. To provide robustness, the methodology is applied iteratively.

Figure 5 shows the evaluation of all image pair connectivity for a terrestrial convergent dataset. Since the algorithm is applied iteratively, a confidence level is computed for each pair according to the outlined methodology.

The proposed methodology is able to highlight a high amount of wrong correspondences, improving the subsequent processing phases and computational time, which is especially relevant for the ASIFT case. However, these results are constrained by the image acquisition protocol and the detector employed, being more susceptible to reject valid pairs in those scenes with high perspective changes (as it is shown in the Tapioca case).

\subsection{Analysis of tie points distribution}

The existence of a high volume of information as a result of the feature extraction and matching process provides computational cost problems during the bundle adjustment process. GRAPHOS allows to generate a homogeneous tie point distribution in the entire image (getting reductions up to $80 \%$ of processing time), generating a more efficient and effective input for the orientation and calibration processes (Figure 6). GRAPHOS offers a friendly interface (Figure 7) to execute and evaluate the algorithm allowing choosing the optimal parameters before the execution of the orientation and self-calibration processes.
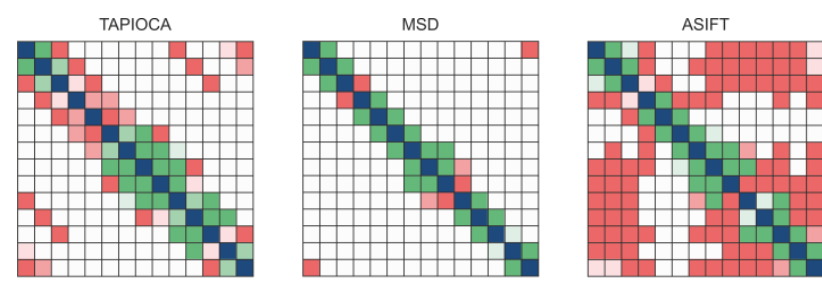

Confidence leve

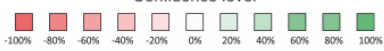

Figure 5. Images connection graphs of a single dataset for the result of all tie point detectors implemented in GRAPHOS.

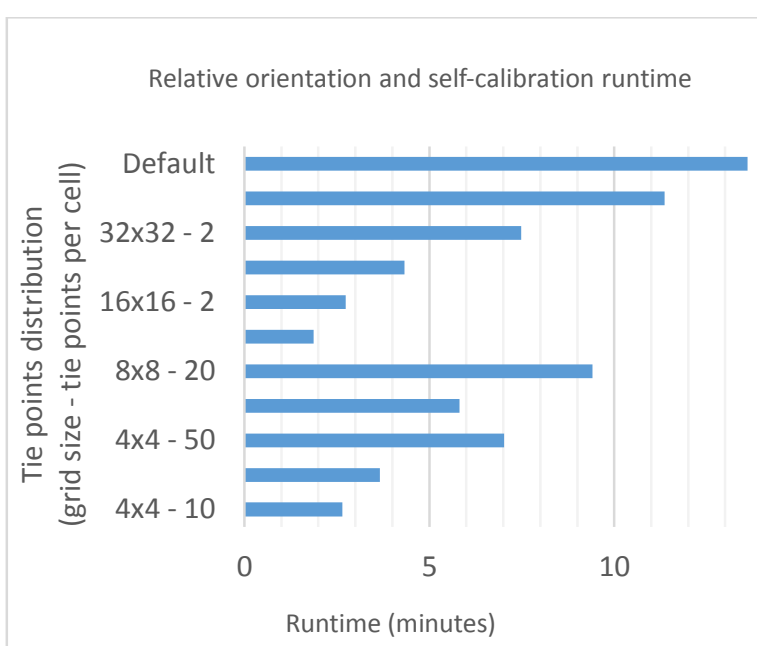

Figure 6. Relative orientation and self-calibration runtime. Example with a dataset of 124 aerial images $(6000 \times 4000$ pixels).

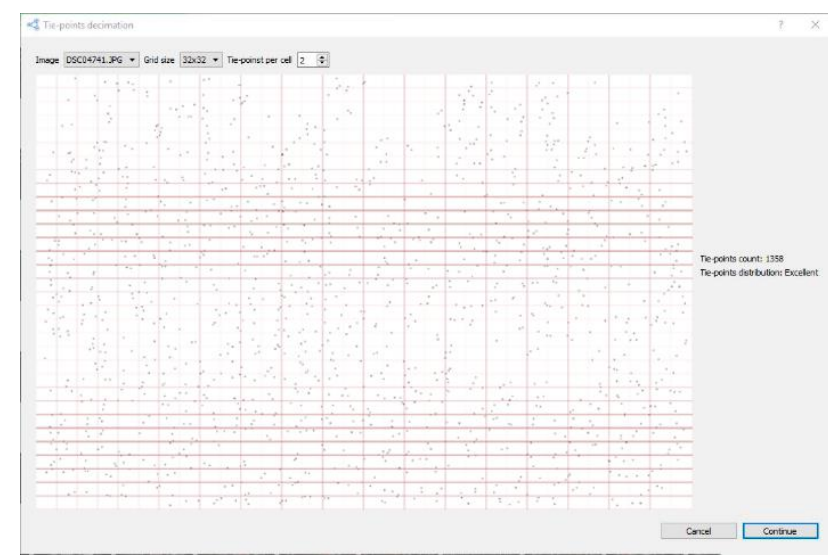

Figure 7. Graphic user interface for the analysis of tie point distribution. Two tie points per cell with a $32 \times 32$ structure.

\subsection{Sensor calibration management}

GRAPHOS includes a tool (Figure 8) to manage different camera calibration formats, allowing to export, import, convert, plot and compare calibration values among the most popular photogrammetric tools (i.e. Photoscan, Photomodeler, etc.).

\subsection{Accuracy assessment and ground truth comparison}

Some statistical parameters related with the photogrammetric bundle adjustment are stored in a log file, including information about sigma0, re-projection error, theoretical precision of object coordinates, etc. In addition, ground truth comparison algorithms have been implemented in order to provide a simple tool (Figure 9) for quality assessment of the results, providing a statistical evaluation of discrepancies. 


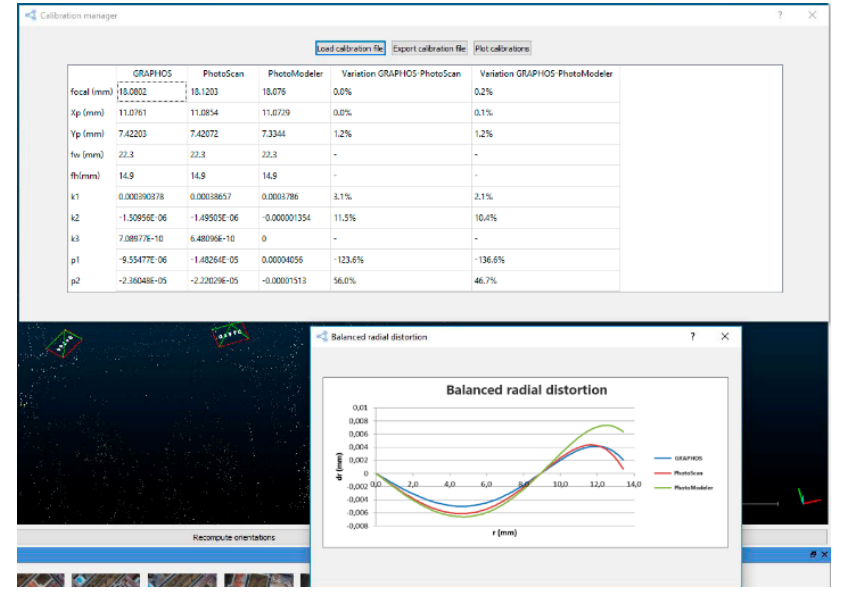

Figure 8. Calibration manager and radial distortion profiles tools.

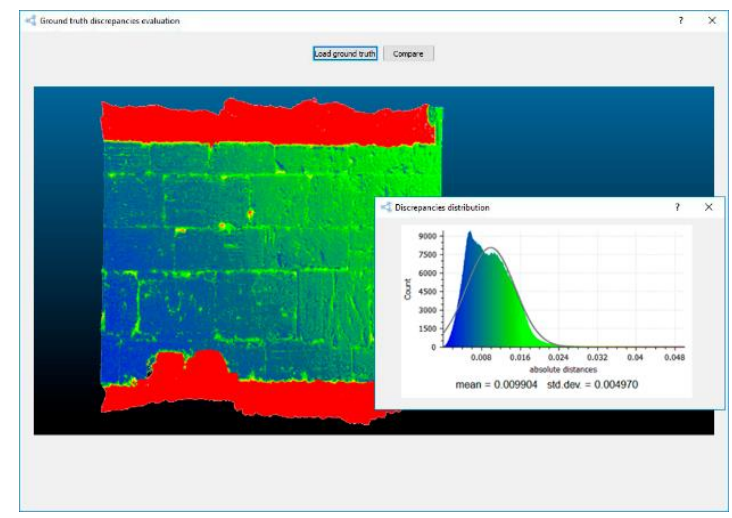

Figure 9. Comparison between the dense matching provided by GRAPHOS and the ground truth obtained with a laser scanner.

\section{CONCLUSIONS AND FUTURE PERSPECTIVES}

This article has reported the developed educational tool named GRAPHOS. It was a joint project among USAL, UCLM, FBK and UNIBO supported by an ISPRS Scientific Initiative. The paper has shown the potential and transversal character offered by the combination of photogrammetry and computer vision procedures in (close-range) architectural, heritage and engineering applications, which usually exhibit geometric and radiometric complexity. The presented tool encloses a strong educational component beyond the most common black-box solutions for 3D image processing and point cloud generation. In particular, some tutorials, simulations and help for advanced parameters have been included in order to support theoretical classes for bachelor and master students, providing also a research platform for $\mathrm{PhD}$ students. The proposed processing methodology guarantees automatism (in the 3D point cloud reconstruction), flexibility (feasible with conventional and noncalibrated cameras or even smartphone sensors) and quality (guaranteeing high accuracy and resolution). A large dataset of images was setup using different sensors and levels of complexity (geometry and radiometry), allowing us to refine and validate the tool. The feedback provided by this dataset allowed us to define the most suitable pipeline or GRAPHO for processing close-range image networks of complex scenarios. Future investigations regarding GRAPHOS will be focused on further optimizing computational cost, especially in orientation and dense matching steps, as well as to cope with larger image dataset. For these reasons, newly Apero-MicMac algorithms and other third party tools like Bundler or DGAP will be evaluated and included in the current implementation. Regarding image pre-processing, feature extraction and matching steps, any innovation introduced by the scientific community will also be analysed and implemented according to their effectiveness and efficiency results.

\section{ACKNOWLEDGEMENTS}

Authors are thankful to ISPRS for supporting GRAPHOS activities and developments through the Scientific Initiative "Advances in the Development of an Open-source Photogrammetric Tool".

\section{REFERENCES}

Agarwal, S., Snavely, N., Seitz, S.M., Szeliski, R., 2010. Bundle adjustment in the large. Proc. ECCV 2010, pp. 29-42.

Apollonio, F., Ballabeni, A., Gaiani, M., Remondino, F., 2014. Evaluation of feature-based methods for automated network orientation. International Archives of Photogrammetry, Remote Sensing and Spatial Information Sciences, Vol. 40(5), pp. 47-54.

Crandall, D.J., Owens, A., Snavely, N., Huttenlocher, D.P., 2013. SfM with MRFs: Discrete-continuous optimization for largescale structure from motion. IEEE Transactions on Pattern Analysis and Machine Intelligence, Vol. 35, pp. 2841-2853.

Deseilligny, M.P., Clery, I., 2011. Apero, an open source bundle adjusment software for automatic calibration and orientation of set of images. International Archives of the Photogrammetry, Remote Sensing and Spatial Information Sciences, Vol. 38(5).

Frahm, J.-M., Fite-Georgel, P., Gallup, D., Johnson, T., Raguram, R., Wu, C., Jen, Y.-H., Dunn, E., Clipp, B., Lazebnik, S., 2010. Building Rome on a cloudless day. Proc. ECCV 2010, pp. 368-381.

Fraser, C., 1980. Multiple focal setting self-calibration of closerange metric cameras. Photogrammetric Engineering and Remote Sensing, Vol. 46, pp. 1161-1171.

Furukawa, Y., Ponce, J., 2010. Accurate, dense, and robust multiview stereopsis. IEEE Transactions on Pattern Analysis and Machine Intelligence, Vol. 32, pp. 1362-1376.

Gaiani, M., Remondino, F., Apollonio, F., Ballabeni, A., 2016. An advanced pre-processing pipeline to improve automated photogrammetric reconstructions of architectural scenes. Remote Sensing, Vol. 8(3).

Gonzalez-Aguilera, D., Gomez-Lahoz, J., 2006. sv3Dvision: didactical photogrammetric software for single image-based modeling. International Archives of the Photogrammetry, Remote Sensing and Spatial Information Sciences, Vol. 36(6), pp. 171-178.

González-Aguilera, D., Guerrero, D., Hernández-López, D., Rodríguez-Gonzálvez, P., Pierrot, M., Fernández-Hernández, J., 2012. PW, Photogrammetry Workbench, CATCON Silver Award.

Gussenmayer, P., Drap, P., 2001. Possibilities and limits of Web photogrammetry-Experiences with the ARPENTEUR web based tool. Proc. Photogrammetric Week, pp. 275-282. 
Hartmann, W., Havlena, M., Schindler, K., 2015. Recent developments in large-scale tie-point matching. ISPRS Journal of Photogrammetry and Remote Sensing, in press.

Heinly, J., Schonberger, J.L., Dunn, E., Frahm, J.-M., 2015. Reconstructing the world* in six days*(as captured by the Yahoo 100 million image dataset). Proc. CVPR, pp. 3287-3295.

Kukelova, Z., Pajdla, T., 2007. A minimal solution to the autocalibration of radial distortion. Proc. CVPR, pp. 1-7.

Lowe, D.G., 1999. Object recognition from local scale-invariant features. Proc. 7th IEEE CV Conf., Vol. 2, pp. 1150-1157.

Lu, C., Xu, L., Jia, J., 2012. Contrast preserving decolorization. IEEE International Computational Photography (ICCP), Conference, pp. 1-7.

Lucas, B.D., Kanade, T., 1981. An iterative image registration technique with an application to stereo vision. International Joint Conference on Artificial Intelligence, pp. 674-679.

Luhmann, T., 2016. Learning photogrammetry with interactive software tool PhoX. International Archives of the Photogrammetry, Remote Sensing and Spatial Information Sciences, Vol. 41, in press.

Maini, R., Aggarwal, H., 2010. A comprehensive review of image enhancement techniques. Journal of Computing, Vol. 2(3).

Morel, J.-M., Yu, G., 2009. ASIFT: A new framework for fully affine invariant image comparison. SIAM Journal on Imaging Sciences, Vol. 2, 438-469.

Muja, M., Lowe, D.G., 2009. Fast Approximate Nearest Neighbors with automatic algorithm configuration. VISAPP (1) 2, 331-340.

Nony, N., De Luca, L., Godet, A., Pierrot-Deseilligny, M., Remondino, F., van Dongen, A., Vincitore, M., 2012. Protocols and assisted tools for effective image-based modeling of architectural elements. LNCS Vol. 7616, pp. 432-439.
Pierrot-Deseilligny, M., Rupnik, E., Girod, L., Belvaux, J., Maillet, G., Deveau, M., Choqueux, G., 2015. MicMac, Apero, Pastis and Other Beverages in a Nutshell. MicMac documentation. Available at: http://logiciels.ign.fr/IMG/pdf/docmicmac-2.pdf.

Remondino, F., Spera, M.G., Nocerino, E., Menna, F., Nex, F., 2014. State of the art in high density image matching. The Photogrammetric Record, Vol. 29, pp. 144-166.

Rothermel, M., Wenzel, K., Fritsch, D., Haala, N., 2012. Sure: Photogrammetric surface reconstruction from imagery, Proceedings LC3D Workshop, Berlin, pp. 1-9.

Snavely, N., Seitz, S.M., Szeliski, R., 2008. Modeling the world from internet photo collections. International Journal of Computer Vision, Vol. 80, pp. 189-210.

Tombari, F., Di Stefano, L., 2014. Interest Points via Maximal Self-Dissimilarities. Proc. ACCV, pp. 586-600.

Verhoeven, G., Karel, W., Stuhec, S., Doneus, M., Trinks, I., Pfeifer, N., 2015. Mind your grey tones - examining the influence of decolourization methods on interest point extraction and matching for architectural image-based modelling. International Archives of Photogrammetry, Remote Sensing and Spatial Information Sciences, Vol. 40(5/W4), pp. 307-314.

Wallis, K.F., 1974. Seasonal adjustment and relations between variables. Journal of the American Statistical Association, Vol. 69 , pp. 18-31.

Wu, C., Agarwal, S., Curless, B., Seitz, S.M., 2011. Multicore bundle adjustment. Proc. CVPR, pp. 3057-3064.

Zancajo-Blazquez, S., Gonzalez-Aguilera, D., Gonzalez-Jorge, H., Hernandez-Lopez, D., 2015. An automatic image-based modelling method applied to forensic infography. PLOS ONE, 10(3): e0118719. 